\title{
Minimal spectral contrast of formant peaks for vowel recognition as a function of spectral slope
}

\author{
ANDREW P. LEA \\ ATR Human Information Processing Laboratories, Kyoto, Japan \\ and \\ QUENTIN SUMMERFIELD \\ MRC Institute of Hearing Research, Nottingham, England
}

\begin{abstract}
In four experiments we investigated whether listeners can locate the formants of vowels not only from peaks, but also from spectral "shoulders"-features that give rise to zero crossings in the third, but not the first, differential of the excitation pattern-as hypothesized by Assmann and Summerfield (1989). Stimuli were steady-state approximations to the vowels [a, i, 3, u, s] created by summing the first 45 harmonics of a fundamental of $100 \mathrm{~Hz}$. Thirty-nine harmonics had equal amplitudes; the other 6 formed three pairs that were raised in level to define three "formants." An adaptive psychophysical procedure determined the minimal difference in level between the 6 harmonics and the remaining 39 at which the vowels were identifiably different from one another. These thresholds were measured through simulated communication channels, giving overall slopes to the excitation patterns of the five vowels that ranged from $-1 \mathrm{~dB} / \mathrm{erb}$ to $+2 \mathrm{~dB} / \mathrm{erb}$. Excitation patterns of the threshold stimuli were computed, and the locations of formants were estimated from zero crossings in the first and third differentials. With the more steeply sloping communication channels, some formants of some vowels were represented as shoulders rather than peaks, confirming the predictions of Assmann and Summerfield's models. We discuss the limitations of the excitation pattern model and the related issue of whether the location of formants can be computed from spectral shoulders in auditory analysis.
\end{abstract}

When two people speak concurrently, a formant in one voice may be partially masked by a more intense formant in the competing voice. The partially masked formant may be displayed as only an irregularity on one of the skirts of the more intense formant in the internal representation of the spectrum. Assmann and Summerfield (1989) called such irregularities shoulders and offered a formal definition, which is restated in the next section. Assmann and Summerfield compared the ability of four models to predict the identification responses made by listeners to pairs of synthetic vowels with the same fundamental frequency $(f 0)$ that were presented concurrently to the same ear. The two models that performed best used shoulders in addition to peaks as evidence of the locations of formants. One explanation of this result is that listeners, like the models, can use shoulders to locate the positions of formants. An alternative explana-

The experiments described in this paper were performed at the Institute of Hearing Research while A.P.L. was supported by a studentship from the Medical Research Council. We wish to thank ATR Human Information Processing Research Laboratories, where this paper was written. Our thanks also go to Joanne Miller, Dianne Van Tasell, and two anonymous reviewers for helpful comments on an earlier version of this paper. Address correspondence to A. P. Lea, ATR Human Information Processing Research Laboratories, 2-2 Hikaridai, Seika-cho, Kyoto 619-02, Japan. tion is that listeners use only peaks to locate formants, but improve their performance by deploying a knowledge of other aspects of the spectral shape of particular vowels. For example, they might know that the spectrum of $[u]$ slopes strongly downward from low to high frequencies, and thus that the second and third formants of [u] are easily masked by energy in a competing vowel. The vowel [i], on the other hand, has more energy in high-frequency regions than [u] does, so its higher formants are more difficult to mask. Assmann and Summerfield's models of vowel identification did not possess this additional knowledge. Possibly, the models compensated by using shoulders as well as peaks to locate formants.

Indirect evidence that listeners can locate formants from shoulders has been provided by Chistovich and Lublinskaya (1979). They determined the position of the phoneme boundary between a single-formant central vowel and a two-formant back vowel by altering the intensity of the first formant $(F 1)$ in relation to a fixed, second formant $(F 2)$ of the back vowel. In some conditions at the phoneme boundary, the $F 1$ was represented by a shoulder rather than by a peak.

The experiments described in the present paper were designed to determine the minimal spectral contrast that is required to specify the location of a formant to listeners with normal hearing. The aim was to provide a direct 
test of the hypothesis that a formant can be specified by a shoulder in the internal representation of a vowel. Two versions of this hypothesis were examined: a "weak" version and a "strong" version. The weak version states that the relevant "internal representation" is the one simulated in the models of Assmann and Summerfield (1989). The strong version states that Assmann and Summerfield's modeling procedure faithfully simulates a representation generated in auditory analysis; thus, listeners can estimate the locations of formants from shoulders in their own internal spectra.

\section{Definitions of Peaks and Shoulders}

Peaks and shoulders are features that can be found in the excitation patterns, or auditory spectra, of sounds. The rationale for using excitation patterns as a basis for understanding aspects of the perception of speech sounds is as follows. An essential property of peripheral auditory analysis is to ensure that energy in different frequency bands generates neural excitation in different fibers of the auditory nerve. In this role as a frequency analyzer, it has been convenient to model the peripheral auditory system as an array of linear, overlapping bandpass filters, with successive filters in the array tuned to different center frequencies (e.g., Helmholtz, 1863/1968; Moore \& Glasberg, 1983b; Patterson, 1974; Zwicker \& Feldtkeller, 1967). The frequency responses of these "auditory" filters have been estimated from the results of masking experiments (see Patterson \& Moore, 1986, for a review), with the result that it is possible to program an array of such filters as a digital auditory filter bank (e.g., Assmann \& Summerfield, 1990; Patterson \& Holdsworth, 1990). The excitation pattern of a sound can be computed by presenting the waveform of the sound to each member of the array of filters and plotting the root-mean-squared levels of the waveforms emerging from the filters as a function of the center frequencies of the filters. In plotting excitation patterns, we have scaled the frequency axis in units of the equivalent rectangular bandwidths (erbs) of the filters. Equal increments along this scale correspond to equal distances of approximately $0.85 \mathrm{~mm}$ along the cochlear partition. For this reason, excitation patterns can be thought of as providing an estimate of the distribution of auditory excitation across place in the peripheral auditory system (Moore \& Glasberg, 1986). Excitation patterns have proved to be more informative than Fourier spectra for understanding many aspects of the perception of complex sounds (e.g., Moore \& Glasberg, 1986), including aspects of the perception of speech (e.g., Assmann \& Summerfield, 1989).

In computing excitation patterns in the present paper, we have used the computational strategy of amplitude weighting in the frequency domain described by Moore and Glasberg (1983b). In implementing the strategy, we included the correction for the variation in absolute sensitivity of human listeners with frequency described by Moore and Glasberg (1987). The correction reduces the excitation level at low and high frequencies.
Panel A of Figure 1 shows the excitation pattern of an [a] vowel, which consisted of the first 45 harmonics of a $100-\mathrm{Hz} f 0$. Initially, the amplitudes of the harmonics were set so that the spectral envelope of the excitation pattern sloped upward from low to high frequencies at $+1 \mathrm{~dB} / \mathrm{erb}$. The amplitudes of 6 harmonics were then raised by $1.4 \mathrm{~dB}$. The frequencies of these 6 harmonics were $600,700,900,1000,2900$, and $3000 \mathrm{~Hz}$; that is, they formed three adjacent pairs. We can consider that the three pairs define the first three formants of a minimalist vowel. The following steps can be used to determine whether the formants are represented as peaks or shoulders. (1) The excitation pattern is sampled at integer multiples of the $f 0$ to produce a smooth spectral envelope, thereby avoiding confusing resolved harmonics
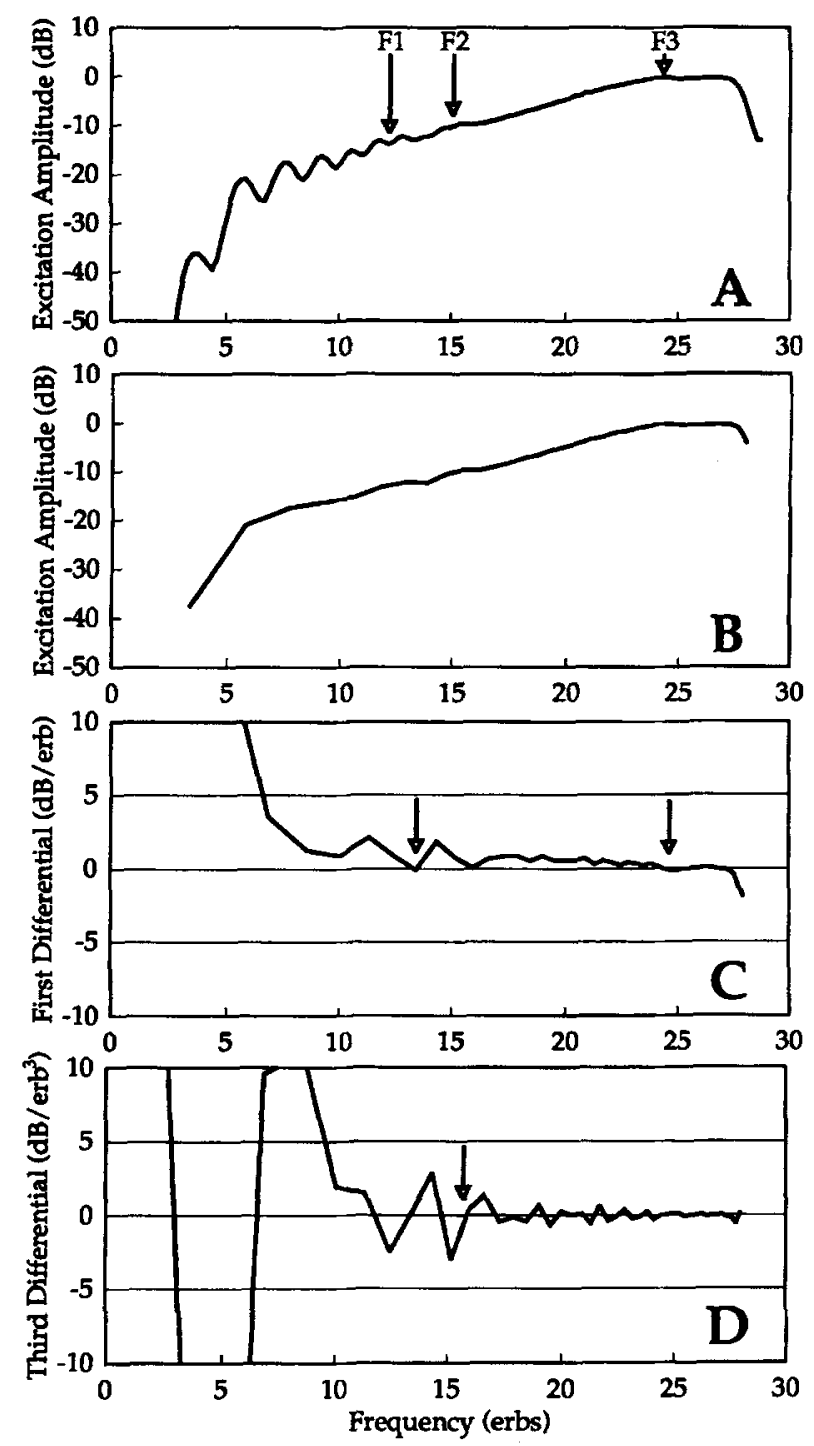

Figure 1. Panel A: excitation pattern of the +1-dB/erb [a] vowel at the average threshold contrast of the four listeners. Panel $B$ : the sampled excitation pattern from $A$. Panel $C$ : the first differential of $B$. Panel D: the third differential of $B$; arrows indicate the zero crossings that were used to locate formants. 
with low-frequency formants. (2) The spectral envelope is differentiated with respect to frequency. "Peaks" in the original excitation pattern are defined as features that appear as negative-going zero crossings in this first differential. (3) The pattern is differentiated twice more. "Shoulders" in the original excitation pattern are defined as features that appear as positive-going zero crossings in this third differential, provided they did not appear as negative-going zero crossings in the first differential.

Panel B of Figure 1 shows the sampled excitation pattern resulting from sampling the excitation pattern in panel A at integer multiples of $100 \mathrm{~Hz}$. Panel C contains the first differential. Panel D contains the third differential. Panel $\mathrm{C}$ contains two negative-going zero crossings; they have been marked by arrows and locate the positions of the first and third formants. The arrow in panel D marks the positive-going zero crossing, which locates the position of the second formant. Thus, in this minimalist vowel, the first and third formants appear as peaks in the original excitation pattern, whereas the second formant appears as a shoulder.

The aim of the present experiments was to determine whether listeners could locate formants from shoulders as well as peaks in minimalist vowels, like the one illustrated in Figure 1. We presented vowel-like sounds with sloping spectral envelopes to listeners for identification to determine just how "minimalist" each vowel could be, and yet be identified correctly. We then determined whether the formants were specified by peaks or shoulders in the excitation pattern of the vowel. It was necessary to use sounds with sloping spectra because a formant can be specified by a shoulder rather than by a peak only when the underlying spectrum is sloping.

\section{GENERAL METHOD}

\section{Stimuli}

Stimuli consisted of two types of sound: a "masker" and a set of "target vowels." Both were generated digitally by additive harmonic synthesis $(10,000 \mathrm{samples} / \mathrm{sec}, 12$-bit amplitude quantization). Each was $400 \mathrm{msec}$ in duration. Onsets and offsets were shaped by the two halves of a $10-\mathrm{msec}$ Hanning window.

The masker consisted of the first 45 harmonics of a $100-\mathrm{Hz} f 0$ with equal amplitudes. Several random-phase spectra were generated for the masker. The one giving the lowest peak factor was chosen and was used for both the masker and the target.

The target vowels were steady-state approximations to five monophthongal vowels of British English, [a], [i], [3], [u], and [o]. Each consisted of six harmonics of $100-\mathrm{Hz} f 0$, chosen to straddle the frequencies of the three formants listed in Table 1. A target vowel was created by retaining six harmonics from the masker and setting the amplitudes of the remaining harmonics to zero. Such stimuli have been used previously (Assmann \& Summerfield, 1989; Leek, Dorman, \& Summerfield, 1987; Summerfield, Sidwell, \& Nelson, 1987).

The stimuli were presented to listeners through five simulated communication channels with different frequency responses and flat phase responses. Their frequency responses were chosen to result in excitation patterns for the maskers with overall slopes of $-1,-0.5,0,+1$, and $+2 \mathrm{~dB} / \mathrm{erb}$, pivoted about the $1-\mathrm{kHz}$ harmonic. Figure 2 shows the excitation pattern of the masker after processing by each of the five communication channels. The
Fourier spectra of the processed maskers had overall slopes that ranged from -5 to $+10 \mathrm{~dB} /$ octave. These values fall within the range of -6 to $+12 \mathrm{~dB} /$ octave, over which Dijkhuizen, Anema, and Plomp (1987) found little variation in speech-reception thresholds for sentences in noise. This implied that normal processes of sentence perception are possible, despite these degrees of overall spectral slope.

\section{Procedure}

Before they were presented to listeners, each target vowel was added to the masker. Remember that the masker contained all of the first 45 harmonics of $100 \mathrm{~Hz}$, but each target contained just 6 of the harmonics. If sine waves of the same frequency and phaseone with a level relative to a reference of $0 \mathrm{~dB}$ and the other with a relative level of $r \mathrm{~dB}$-are summed together, the relative level of the sum is $20 \log _{10}\left(10^{(r / 20)}+1\right)$. Thus, when a target was added to the masker without attenuation, the levels of the 6 harmonics in the resulting composite of masker plus target were $6 \mathrm{~dB}$ more intense than the other 39 harmonics. The "spectral contrast" of each formant in the composite was defined as the difference in level between the 2 harmonics that define each formant and the harmonics of the masker. Hence, when a target was added to the masker without attenuation, the contrast for all three formants would be $6 \mathrm{~dB}$. The case is illustrated in panel A of Figure 3, which shows the amplitude spectrum that results when the target [3] is added to the masker. The aim of the psychophysical procedure was to adjust the intensity of the targets relative to the masker in order to determine the minimal spectral contrast required for each vowel to be identifiably different from the other four vowels.

The procedure was an adaptive two-interval, five-alternative forced-choice task (2I5AFC). A trial consisted of two intervals. The masker was presented in both intervals. In one interval, chosen at random, one of the five targets was also presented. This procedure was originally used by Summerfield and Assmann (1991). The listener's task was to indicate which interval contained the target and what its identity was by pressing visual display unit (VDU) keys labeled with the orthographic representations of the five vowels. After each response, the VDU indicated to the listener which vowel had been presented in which interval.

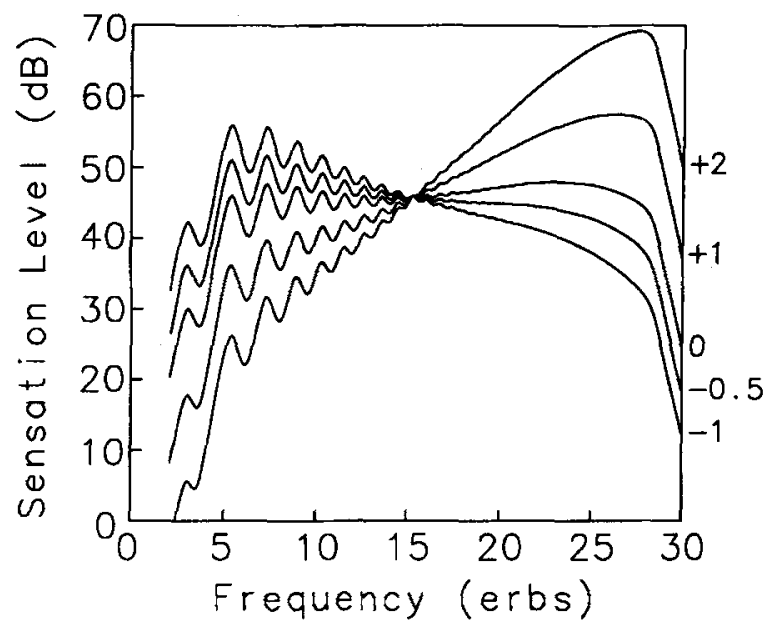

Figure 2. Excitation patterns of the masker after processing to simulate the effects of the five communication channels. The 1-kHz harmonic was the pivotal frequency. The amplitudes of the harmonics have been adjusted in accordance with the variation in absolute sensitivity with frequency, so that $0 \mathrm{~dB}$ represents the absolute pure-tone threshold of listeners with normal hearing. The numbers on the right indicate the slope of the communication channels in decibels per erb. 

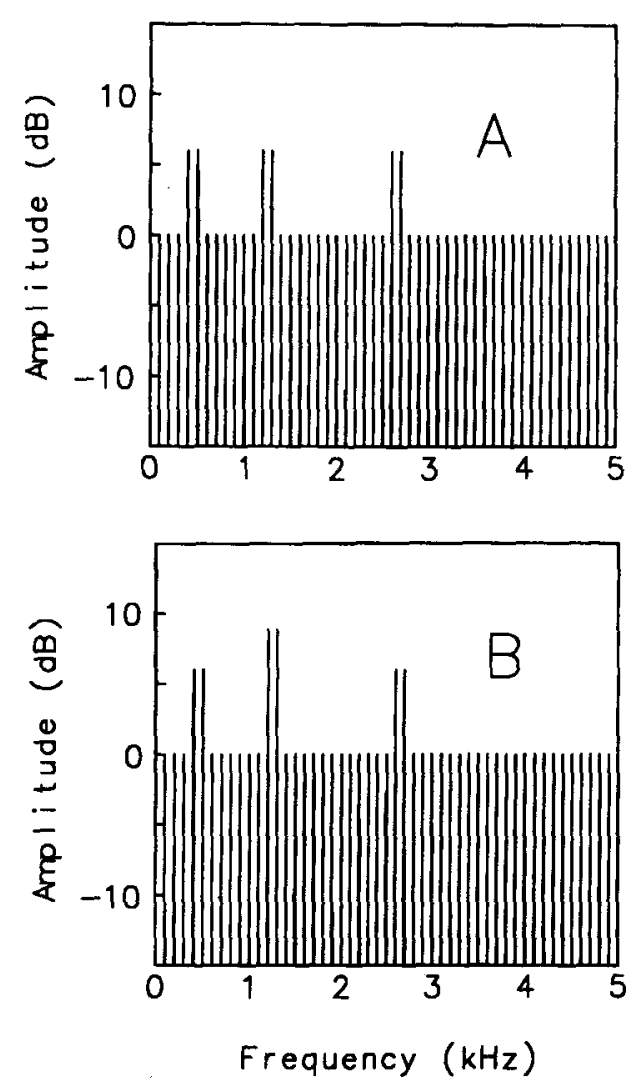

Figure 3. Panel A: Fourier spectra of the masker and the [3] target summed without attenuation, giving formants with $6 \mathrm{~dB}$ of spectral contrast. Panel B: spectrum as in A, but with the harmonics defining the $F 2$ of the target boosted by $5 \mathrm{~dB}$, giving a second-formant peak with $8.9 \mathrm{~dB}$ of spectral contrast.

Five adaptive staircases were run concurrently, one for each target vowel. Trials from the five runs were interleaved randomly. At the start of each run, the level of the targets was higher than the level of the masker, so it was easy for the subjects to identify the target vowels correctly. After two correct responses, the relative level of the targets was reduced by one step. After an incorrect response, the level was raised by one step. This procedure tracks the level giving $71 \%$ correct responses (Levitt, 1971). A change from increasing to decreasing relative level, or vice versa, constituted a reversal. Runs started with a step size of $8 \mathrm{~dB}$, which was reduced to $2 \mathrm{~dB}$ after three reversals. Runs continued until all five staircases had completed (at least) 10 reversals with the smaller step size. The relative level at the last 10 reversals in each run was averaged to provide an estimate of the threshold relative level for each target vowel. By knowing the relative level at threshold, it was possible to compute the amount of contrast in the composite stimulus at threshold. Overall estimates of the threshold contrast for each vowel were obtained by averaging the threshold contrasts measured in four separate runs.

\section{Presentation Levels}

Stimuli were presented on line (Tandon PC-AT) through a CED1401 interface. They were lowpass filtered at $4250 \mathrm{~Hz}$ (Kemo VBF8, -135 dB/octave), attenuated, mixed, and presented through the left headphone of a pair of Sennheiser HD $414 \mathrm{X}$ headphones.
Presentation levels were measured by placing the earphone onto a Brüel \& Kjaer artificial ear (Type 4153) with a flat-plate adapter (Type DB0843). A 150-g weight was used to compress the foam earpad by a force similar to that exerted by the headband of the Sennheiser headphones when worn by a typical adult listener. The sound level was measured by using a .5-in. microphone (Type 4134) connected to a sound-level meter (Type 2235) on its fast setting.

The 1-kHz component of the masker was presented at the same level of $46 \mathrm{~dB}(\mathrm{~A})$ SPL after transmission through each communication channel. The overall levels of the masker, after transmission through the five communication channels $(-1,-0.5,0,+1$, and $+2 \mathrm{~dB} / \mathrm{erb})$, were $52,50,51,58$, and $67 \mathrm{~dB}(\mathrm{~A}) \mathrm{SPL}$.

\section{Listeners}

The 4 listeners, of whom the first author was one, were native speakers of British English. Their ages ranged from 21 to 26 years. Their audiometric thresholds were within $15 \mathrm{~dB}$ of the ANSI (1969) standard at audiometric frequencies between 250 and $8000 \mathrm{~Hz}$ in both ears.

Each listener received from 2 to $6 \mathrm{~h}$ of practice in the vowelidentification task before the data were collected. This amount of practice largely stabilized performance. However, thresholds continued to fall slightly over the course of the four experiments. The experiments were run in the order in which they are reported here, except that Experiment 2 was run before Experiment 1. The effects of ordering and practice are discussed in relation to the results of individual experiments.

In addition to general practice in the vowel-identification task, the listeners completed a practice run for each condition of each experiment before completing four experimental runs, for which data are reported.

\section{EXPERIMENT 1}

Following the work of Joos (1948) and Delattre, Liberman, Cooper, and Gertsman (1952), it is generally accepted that the frequencies of the first and second formants, and occasionally the third formant, are required to specify the phonetic identity of vowels unambiguously. However, Table 1 shows that, potentially, the five target vowels could be distinguished solely from their different $F 2$ s. Experiment 1 was run as a preliminary test to establish which formants are used by listeners to identify the target vowels. Such evidence is important for interpreting the results of subsequent experiments because it would not be valid to conclude that listeners could use a shoulder to locate a formant when identifying a vowel, if that formant was not in fact used in identifying the vowel.

Table 1

Frequencies (in Hertz) of the Three Formants Used to Synthesize the Five Vowels

\begin{tabular}{cccccc}
\hline & \multicolumn{5}{c}{ Vowels } \\
\cline { 2 - 6 } Formants & {$[\mathrm{a}]$} & {$[\mathrm{i}]$} & {$[3]$} & {$[\mathrm{u}]$} & {$[\mathrm{0}]$} \\
\hline$F 1$ & 650 & 250 & 450 & 250 & 350 \\
$F 2$ & 950 & 2250 & 1250 & 850 & 750 \\
$F 3$ & 2950 & 3050 & 2650 & 1950 & 2850 \\
\hline
\end{tabular}

Note-These formant frequencies have been used previously (Assmann \& Summerfield, 1989; Leek et al., 1987; Summerfield et al., 1987). Each formant was defined by two harmonics placed $50 \mathrm{~Hz}$ on either side of the formant frequency. 


\section{Method}

Only the communication channel with a slope of $+1 \mathrm{~dB} / \mathrm{erb}$ was used. Four conditions were run. For the first condition, the original targets were used; the six harmonics had equal amplitudes. For the second condition, a new set of targets was created by boosting by $5 \mathrm{~dB}$ the levels of the pairs of harmonics defining the $F 1 \mathrm{~s}$ of the vowels. For the third condition the $F 2 \mathrm{~s}$ were boosted by $5 \mathrm{~dB}$, and for the fourth condition the $F 3$ s were boosted by $5 \mathrm{~dB}$. Note that boosting a formant by $5 \mathrm{~dB}$ does not increase its contrast by this amount after the target vowel has been added to the masker. Comparison of panels $\mathrm{A}$ and $\mathrm{B}$ of Figure 3 shows that boosting $\mathrm{F} 2$ by $5 \mathrm{~dB}$ increases the contrast of $F 2$ by only $2.9 \mathrm{~dB}$ when the target vowel is added to the masker without attenuation. ${ }^{l}$ With attenuation, the increase in contrast caused by boosting is further reduced.

The rationale for the experiment was as follows. Suppose that a listener performs the 2I5AFC task by determining the frequency of only $F 2$, ignoring $F 1$ and $F 3$. In this case, the contrast of $F 2$ at threshold will be constant across the four conditions, as only $F 2$ is being used by the listener. In particular, the contrast of $F 2$ at threshold will be the same for the condition in which $F 2$ is boosted and for the baseline condition. Conversely, if the listener performs the task by discriminating the frequency of only $F 1$, contrast of $F 2$ at threshold will be greater in the condition in which $F 2$ is boosted than in the baseline condition, as the threshold of the listener depends upon the contrast of $F 1$, not $F 2$. Thus, the formant's contribution to the vowel-identification task can be estimated by determining the extent to which threshold contrast is increased, relative to the baseline, when that formant is boosted. If the contrast is greatly increased, the formant makes little contribution.

\section{Results}

The results of Experiment 1 are summarized in Figures 4 and 5 . Figure 4 shows the mean contrast at threshold in each condition for the five vowels individually, averaged over the 4 listeners. Figure 5 shows results for the 4 individual listeners, and their mean, averaged over the five vowels. The error bars for individual subjects in Figure 5 show plus and minus one standard deviation $(S D)$. In accordance with the principles of repeated measures analyses (e.g., Winer, 1971), in which effects of treatments for a subject are measured relative to the average score produced by that subject across all treatments, the error bars in Figure 4 (and in the panel of Figure 5 that shows the mean data) plot plus and minus one "intralistener" standard deviation (ILSD)-that is, the standard deviation of thresholds computed after subtracting the mean threshold of each subject from his/her individual thresholds.

In Figures 4 and 5, the leftmost point in each panel shows contrast at threshold in the baseline condition, where none of the formants were boosted. The lower horizontal line, which intersects this point, indicates the threshold that would occur if a formant were boosted and if the contrast of that formant alone determined the contrast at threshold. The upper horizontal line indicates the threshold that would be found if the boosted formant made no contribution. This upper horizontal line was estimated by taking the formant contrast for the baseline condition and calculating the increase in formant contrast that would be produced by adding $5 \mathrm{~dB}$ to the am-

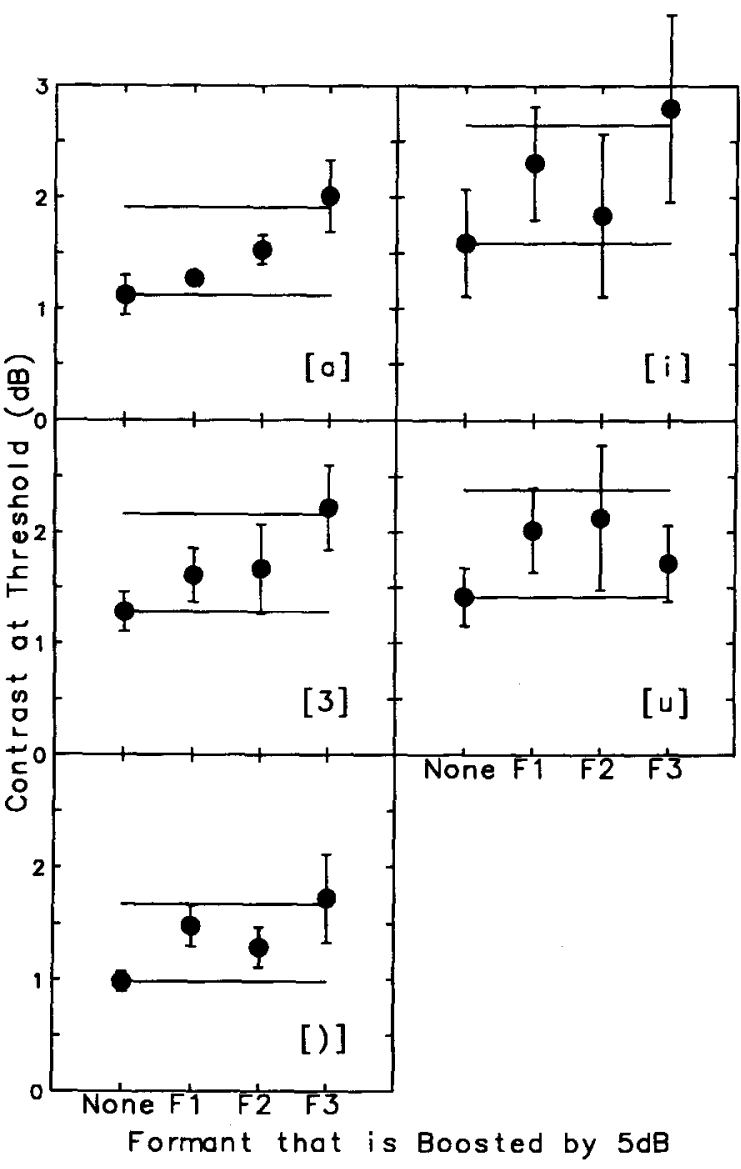

Figure 4. Results of Experiment 1 plotted as thresholds for each vowel, averaged over the 4 listeners. Results for the four conditions are arrayed from left to right and labeled according to which of the formants was boosted in level. Error bars extending beyond the plotting symbol show $\pm 1 I L S D$ (see text). The lower horizontal lines indicate the threshold that would occur when a formant is boosted if that formant alone controlled performance. The upper horizontal lines indicate the threshold that would be found if the formant made no contribution.

plitude of the formants at synthesis. The data were examined in a repeated measures analysis of variance (ANOVA) with the factors of experimental condition (which formant was boosted) and vowel. There was a significant effect of condition $[F(3,9)=7.25, p<.009]$ and a significant interaction between experimental condition and vowel $[F(12,36)=2.60, p<.01]$, but no main effect of vowel $[F(4,12)=2.30]$. Post hoc tests carried out according to the criteria recommended by Scheffé showed that the mean contrast at threshold was higher when $F 1$ and $F 2$ were boosted than when no formants were boosted, but was lower than when $F 3$ was boosted $(p<.01$ in each case $)$.

The mean results (Figure 5) averaged over listeners and vowels show that, overall, $F 1$ and $F 2$ controlled performance, but $F 3$ made little contribution. Figure 4 


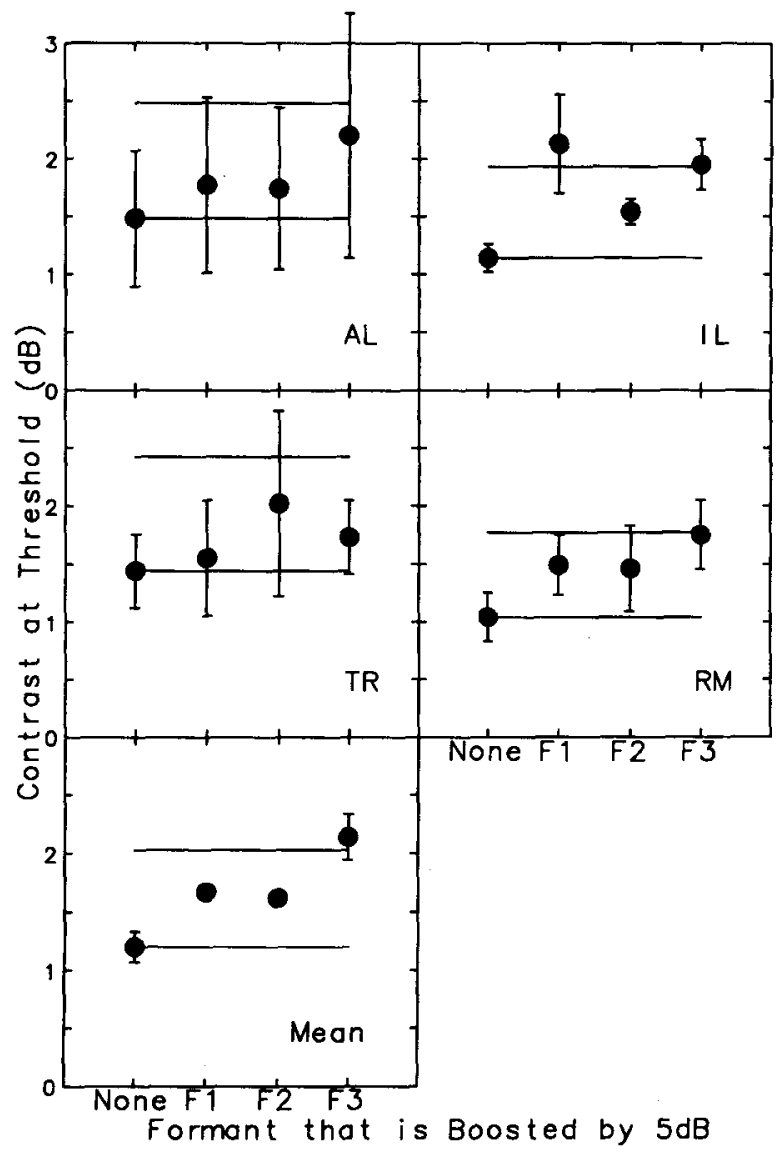

Figure 5. Results of Experiment 1 plotted as thresholds for each listener and their means, averaged over the five vowels. Results for the four conditions are arrayed from left to right and labeled according to which of the formants was boosted in level. Error bars extending beyond the plotting symbol show $\pm 1 S D$ for individual listeners and \pm 1 ILSD (see text) for their average. The lower horizontal lines indicate the threshold that would occur when a formant is boosted if that formant alone controlled performance. The upper horizontal lines indicate the threshold that would be found if the formant made no contribution.

shows that this pattern was shown by [a], [i], [3], and [0]. With [u], all three formants played a role. Figure 5 shows that Listeners A.L. and R.M. displayed the pattern shown in the mean data, whereas T.R. used both $F 1$ and $F 3$, and I.L. mainly used $F 2$.

\section{Discussion}

The baseline threshold contrast of $1.2 \mathrm{~dB}$ is somewhat lower than estimates of about $2 \mathrm{~dB}$ obtained with other methodologies (Leek et al., 1987; Summerfield et al., 1987; Turner \& Van Tasell, 1984). However, it is similar to the estimate of $1.3 \mathrm{~dB}$ reported by Henn and Turner (1990), who measured the minimum detectable increment in the intensity of the $1-\mathrm{kHz}$ component of a series of harmonics of $200 \mathrm{~Hz}$ with otherwise equal intensities. Thus, the minimal spectral contrast that defines a formant is close to the minimum that can be detected.
Although there were some individual differences, overall, the listeners used both $F 1$ and $F 2$ to perform the vowel-identification task. Thus, in the following experiments we sought to determine whether either or both of these formants could be defined as shoulders rather than as peaks at threshold when stimuli were presented through communication channels with steep spectral slopes.

\section{EXPERIMENT 2}

Experiment 2 was designed to test the hypothesis that formants can be specified by shoulders in the excitation pattern of a vowel. Thresholds were measured separately; each of the five communication channels used target vowels with no formants boosted. The five threshold stimuli estimated with each communication channel were then synthesized, and their excitation patterns were examined to establish whether formants were defined by peaks or shoulders.

Listeners attended five 1-h sessions, during which they received a practice run with stimuli presented through each of the five communication channels. This was followed by 4 test runs with each channel, for a total of 20 test runs. During the test runs, the listeners experienced the different communication channels in a randomly chosen order.

\section{Results}

The mean contrast at threshold, averaged over the 4 listeners, is plotted in Figure 6 for each of the five target vowels. The abscissa plots the spectral slope of the communication channel in terms of the slope that it imposes on the excitation pattern of the masker. Error bars extending beyond the plotting symbols show \pm 1 ILSD. The data were examined by using a repeated measures ANOVA with the factors of communication channel slope and vowel. There was a significant effect of slope $[F(4,12)=10.09, p<.008]$, but there was no effect of vowel $[F(4,12)=2.56]$ and no interaction between slope and vowel $[F(16,48)=1.76]$.

Figure 7 shows the mean contrast at threshold for each listener and their mean, averaged over the five target vowels. Error bars for the individual listeners plot $\pm 1 S D$. For the listeners' means, error bars extending beyond the plotting symbols show \pm 1 ILSD. For the averaged data, post hoc tests were carried out according to the criteria recommended by Scheffé. These showed that the threshold at $+2 \mathrm{~dB} / \mathrm{erb}$ was significantly higher than the other four thresholds $(+1 \mathrm{~dB} / \mathrm{erb}, p<.05$; $0 \mathrm{~dB} / \mathrm{erb}, p<.01 ;-0.5 \mathrm{~dB} / \mathrm{erb}, p<.01 ;-1 \mathrm{~dB} / \mathrm{erb}, p<$ $.05)$, which did not differ from each other.

\section{Discussion}

For the 0-dB/erb communication channel, the listeners needed an average formant contrast of $1.5 \mathrm{~dB}$ at threshold. This figure is larger than the $1.2 \mathrm{~dB}$ of contrast needed for the $0-\mathrm{dB} / \mathrm{erb}$ communication channel in Experiment 1, in which no formant was boosted. There are two possible reasons for the larger threshold contrast 


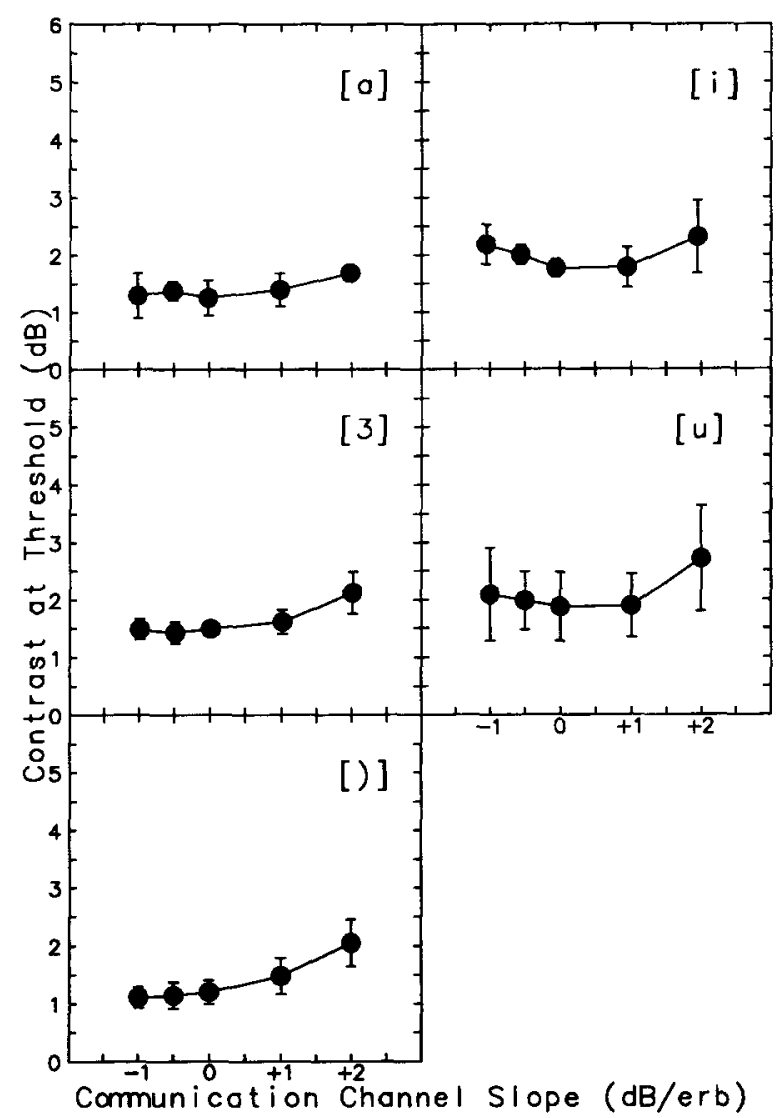

Figure 6. Results of Experiment 2 plotted as thresholds for each vowel averaged over listeners. The abscissa shows the slope of the five communication channels. Error bars extending beyond the plotting symbol show \pm 1 ILSD.

in Experiment 2. First, Experiment 2 was performed before Experiment 1. Therefore, the listeners would have been more familiar with the stimuli and the task when they performed Experiment 1. Second, all four conditions of Experiment 1 used the same communication channel slope, so the listeners were accustomed to it. In contrast, in Experiment 2, the slope varied from run to run within each experimental session.

The 25 stimuli ( 5 communication channels $\times 5$ target vowels) corresponding to the listeners' mean thresholds were synthesized. Excitation patterns were computed from the 400-msec duration of each stimulus. Peaks and shoulders were located by using the procedures described in the introduction and shown in Figure 1 (which shows the analysis of a $+1-d B / e r b$ [a] vowel synthesized at the mean threshold of the listeners). Table 2 summarizes the results of applying the analysis. There are three entries for each vowel and slope, corresponding to $F 1$, $F 2$, and $F 3$, from left to right, respectively. The symbol "-" indicates that the formant was specified by a peak. The "S" indicates that the formant was specified by a shoulder. In relating a spectral feature to a formant, a tolerance of $\pm 50 \mathrm{~Hz}$ was permitted between the nominal formant frequency and the frequency of the spectral feature. Table 2 shows that with extreme slopes of $-1 \mathrm{~dB} / \mathrm{erb}$ and $+2 \mathrm{~dB} / \mathrm{erb}$, some formants were specified by shoulders rather than by peaks.

Table 2 shows that vowel identification generally requires formants to be specified as spectral peaks at threshold. However, when the frequency response of the communication channel has a steep slope, some formants of some vowels need only be specified as shoulders. This result could mean that listeners can interpret shoulders as evidence of formants. However, an alternative possibility is that the listeners used the strategy of spectral subtraction (see Summerfield \& Assmann, 1991) to convert shoulders into peaks. According to this idea, on each trial of the 2I5AFC procedure, listeners do two things: (1) They subtract the internal spectrum of the masker from the internal spectrum of the masker plus target to compute a "difference pattern," and (2) they treat peaks in the difference pattern as formants.

The spectral subtraction strategy is illustrated in Figure 8. Here the masker is subtracted from the [3] vowel plus masker composite for the +2 - $\mathrm{dB} /$ erb communication channel (in which all formants are defined by

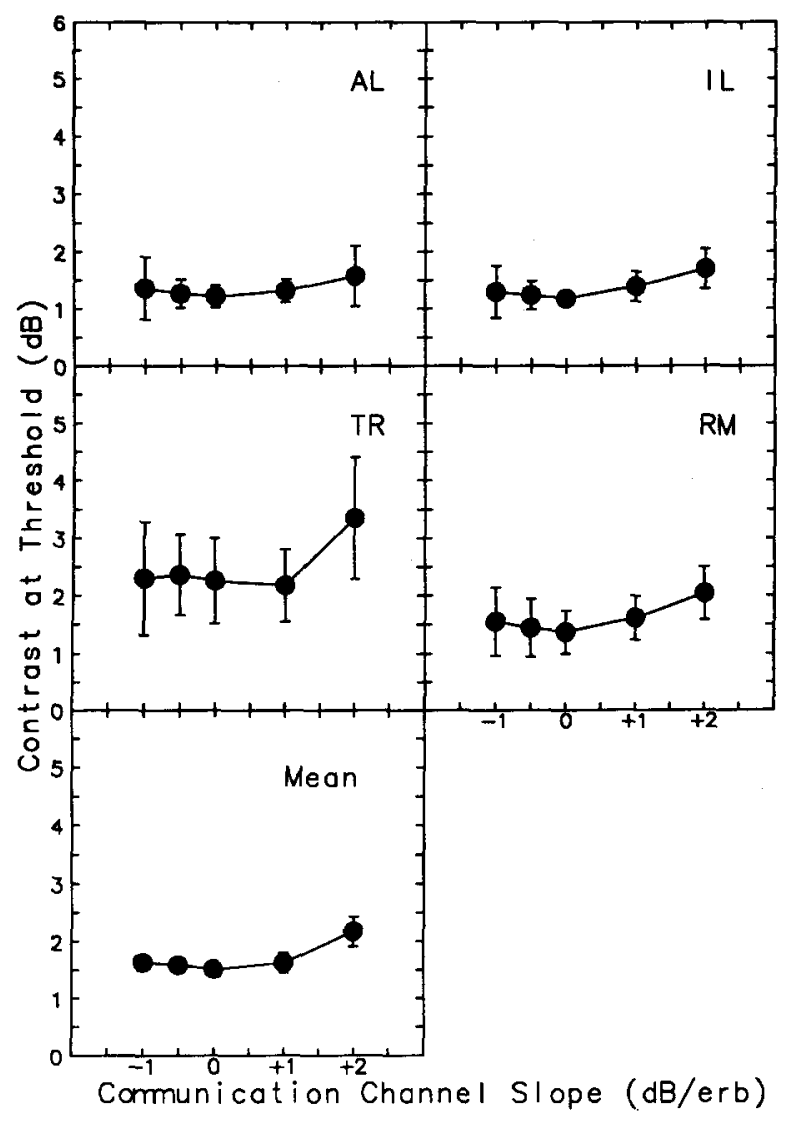

Figure 7. Results of Experiment 2 plotted as thresholds for each listener averaged over vowels. The abscissa shows the slope of the five communication channels. Error bars extending beyond the plotting symbol show $\pm 1 S D$ for individual listeners and $\pm 1 I L S D$ for their mean. 
Table 2

Results of Peak and Shoulder Analysis From Experiment 2

\begin{tabular}{|c|c|c|c|c|c|}
\hline \multirow[b]{2}{*}{ Vowels } & \multicolumn{5}{|c|}{ Communication Channel Slope } \\
\hline & $-1 \mathrm{~dB} / \mathrm{erb}$ & $-0.5 \mathrm{~dB} / \mathrm{erb}$ & $0 \mathrm{~dB} / \mathrm{erb}$ & $+1 \mathrm{~dB} / \mathrm{erb}$ & $+2 \mathrm{~dB} / \mathrm{erb}$ \\
\hline [a] & SSS & $\cdots$ & $\cdots$ & $-\mathrm{S}-$ & SSS \\
\hline [i] & $\cdots$ & $\ldots$ & -..- & --- & SS- \\
\hline$[3]$ & S-- & -.- & -.. & --- & SSS \\
\hline$[u]$ & $\ldots$ & $\cdots$ & $\ldots$ & $-\cdots$ & S-S \\
\hline$[0]$ & -SS &.-- & $\ldots$ & $\ldots$ & SSS \\
\hline
\end{tabular}

Note--Peaks are indicated by "-" and shoulders by S. The left "-" or $S$ refers to $F 1$, the center "-" or S refers to $F 2$, and the right "-" or $S$ refers to $F 3$.

shoulders in Table 2), to leave the difference pattern, in which all formants are defined by peaks.

If the listeners used this strategy, and if the auditory system were linear, vowel identification would not vary with the slope of the communication channel. Figures 6 and 7 show that thresholds do vary with slope, suggesting that listeners may not use spectral subtraction. However, other factors, including the deterioration of frequency selectivity at high intensities (Lutfi \& Patterson, 1984; Weber, 1977) and the difficulty of detection near absolute threshold, may raise thresholds when the slope is extreme. Thus, it is not possible to tell from the results of Experiment 2 whether listeners use shoulders directly to locate formants, or, alternatively, whether they use spectral subtraction to convert shoulders into peaks before locating formants. However, if they use the latter strategy, their performance should be disrupted if they are prevented from benefiting from spectral subtraction. Experiment 3 was a test of this hypothesis.

\section{EXPERIMENT 3}

In Experiment 2, thresholds for each slope were estimated in separate runs. In other words, within a run, the listeners experienced only one communication channel slope. The slope of the masker in the interval of each trial that contained the signal was the same as that in the nonsignal interval. As a result, spectral subtraction was a viable strategy for recovering the spectral shape of the target vowel. In Experiment 3, however, there were four conditions in which the amplitude level and/or the slope of the masker could vary randomly between the signal and nonsignal intervals of each trial. In Condition 1, neither amplitude level nor slope varied. In Condition 2, amplitude level was fixed, but slope varied randomly (among the five slopes). In Condition 3, slope was fixed, but amplitude level varied randomly within a range of $\pm 5 \mathrm{~dB}$. In Condition 4 , both level and slope varied.

Spectral subtraction would be a beneficial strategy in Condition 1, and it might be of some use in Condition 3 if listeners can subtract the spectral profiles of the signals in the two intervals disregarding their levels, but it would be of no use in Conditions 2 and 4 . Thus, evidence that listeners can use spectral subtraction would be found if thresholds in Conditions 1 and 3 were lower than those in the other two conditions.

Each listener participated in five 1-h sessions, during which they first completed a practice run in each of the four conditions. This was followed by four test runs in each condition, which were presented in a random order.

\section{Results}

The mean contrast at threshold in each condition, averaged over vowels and listeners, is plotted in Figure 9. The letters beneath each point indicate whether level (L), slope (S), both, or neither varied randomly within trials. A repeated measures ANOVA showed that the thresholds differ significantly $[F(3,9)=4.7, p<.05]$. Post hoc tests carried out according to the criteria recommended by Scheffè showed that thresholds were significantly higher in Condition 4 than in Condition $1(p<$ .05 ) and that no other conditions differed significantly.

\section{Discussion}

Thresholds were significantly higher in Condition 4, in which both slope and level varied, than in Condition 1 , in which neither slope nor level varied. This outcome suggests that the listeners made some use of the strategy of spectral subtraction in Condition 1 and suffered from the inability to benefit from it in Condition 4. It is possible, therefore, that the listeners used the strat-
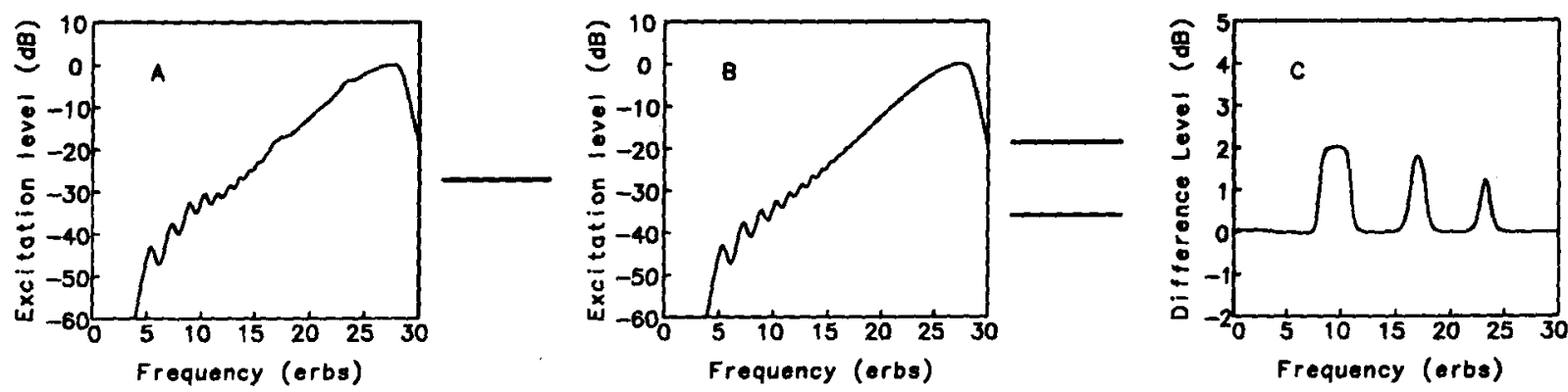

Figure 8. Illustration of the spectral subtraction strategy. Panel A: excitation pattern of the [3] target at the mean threshold of the listeners in the condition with +2-dB/erb communication channel. All three formants are defined as shoulders. Panel B: excitation pattern of the masker alone. Panel C: the difierence pattern that results from subtracting B from A. In C, all three formants are defined by peaks. 


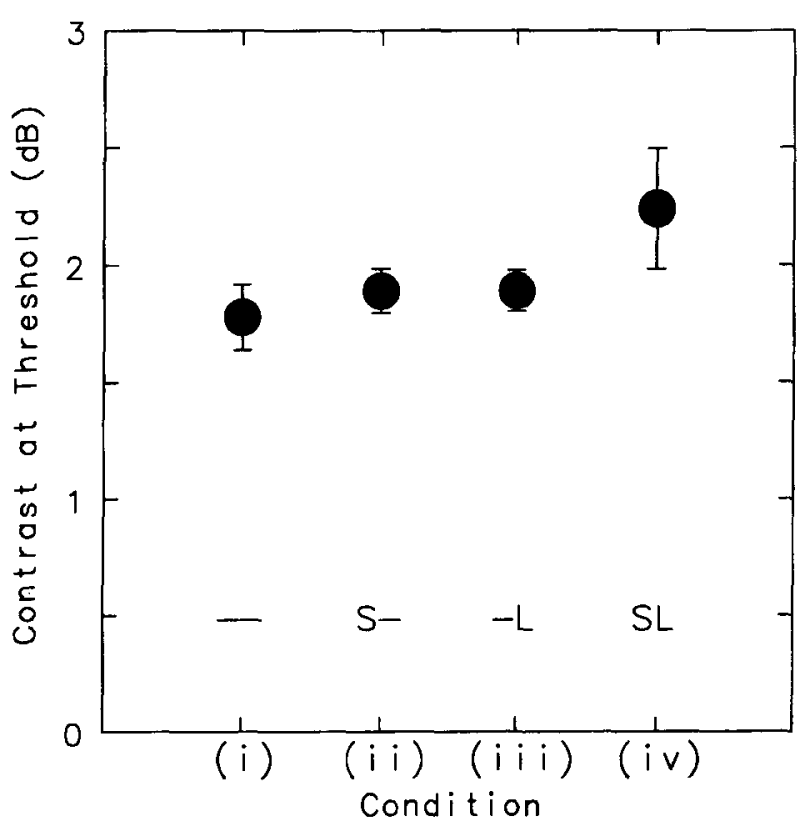

Figure 9. Results of Experiment 3 plotted as thresholds for the average of listeners and vowels. Results for the four conditions are arrayed from left to right and labeled according to whether slope (S), level (L), or neither (-) varied in level. Error bars that are langer than the plotting symbols show \pm 1 ILSD.

egy in Experiment 2 to convert shoulders in the excitation pattern of stimuli into peaks in the difference pattern. Although the mean thresholds plotted in Figure 9 differ significantly, they differ by only a small amount. The similarity suggests that spectral subtraction did not make a major contribution to the listeners' performance and that most of the time they identified the target vowels directly.

Condition 3, in which level varied but slope was fixed, is similar to the conditions of many experiments on profile analysis (Green, 1988). In these experiments, listeners detect increments to the level of one component of a multicomponent complex in conditions in which overall level is varied randomly within trials. In such experiments, experienced listeners produce thresholds corresponding to a spectral contrast as low as $1.4 \mathrm{~dB}$ (Green, Kidd, \& Picardi, 1983). Thresholds in Condition 3 were $1.7,1.5,2.7$, and $2.0 \mathrm{~dB}$ for Listeners A.L., I.L., T.R., and R.M., respectively. Thus, some of the subjects in the present experiments were able to locate formants from degrees of contrast in the spectral profile of a vowel that were close to the minimum amount that can be detected.

It is not possible to establish whether formants at threshold were specified by shoulders or by peaks in Experiment 3 because a "composite" threshold was obtained for each slope, averaged across the five vowels. Accordingly, Experiment 4 was designed to estimate thresholds for each vowel separately with each slope in a condition in which spectral subtraction would not be beneficial.

\section{EXPERIMENT 4}

In this experiment, communication channel slope and level were varied randomly between the intervals of the forced-choice procedure, as in Condition 4 of Experiment 3 . However, 25 adaptive procedures were interleaved to allow a separate threshold to be estimated for each vowel with each communication channel. With 25 independent staircases, each run required about $1 \mathrm{~h}$. Listeners completed a practice run followed by four test runs in five separate sessions.

\section{Results}

The filled circles in Figure 10 plot the mean thresholds for each vowel as a function of slope averaged across listeners. The filled circles in Figure 11 plot the mean thresholds for each listener as a function of slope averaged over the five vowels. The open circles in each figure show the analogous results from Experiment 2, in

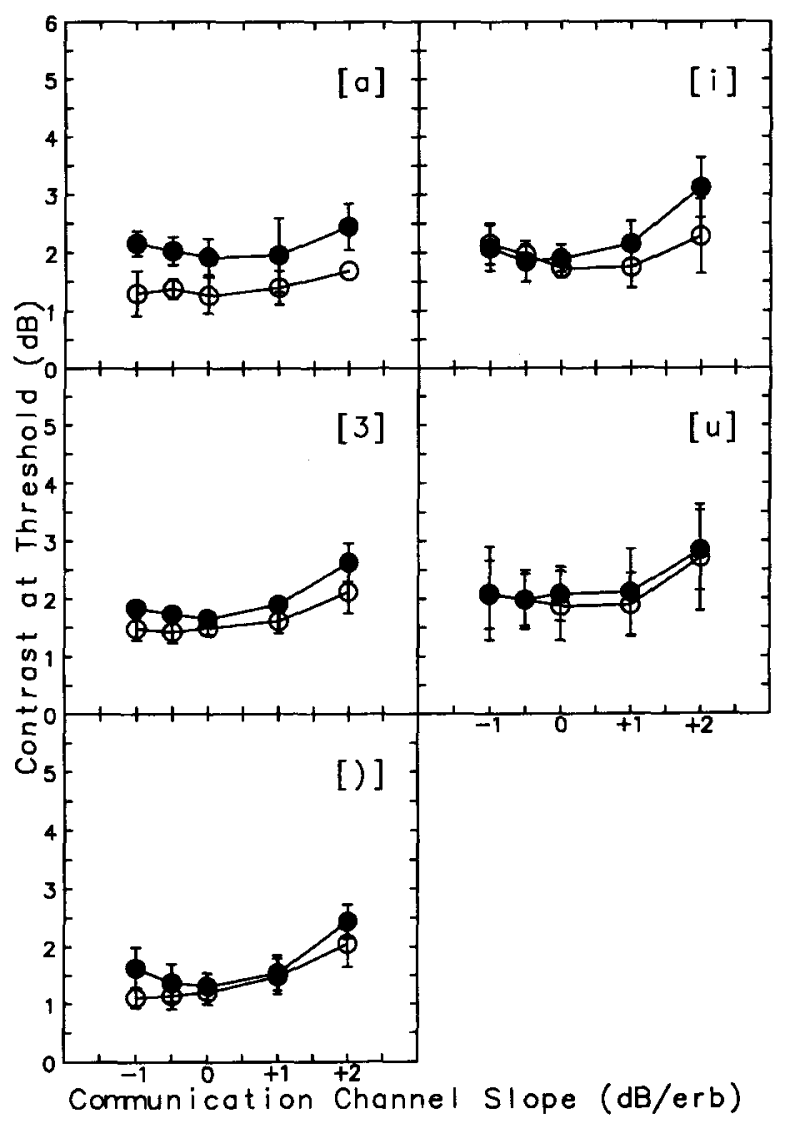

Figure 10. The filled symbols plot the results of Experiment 4 as thresholds for each vowel averaged over listeners. The abscissa shows the slope of the five communication channels. Error bars extending beyond the plotting symbol show \pm 1 ILSD. The open symbols show the analogous results from Experiment 2 for comparison. 


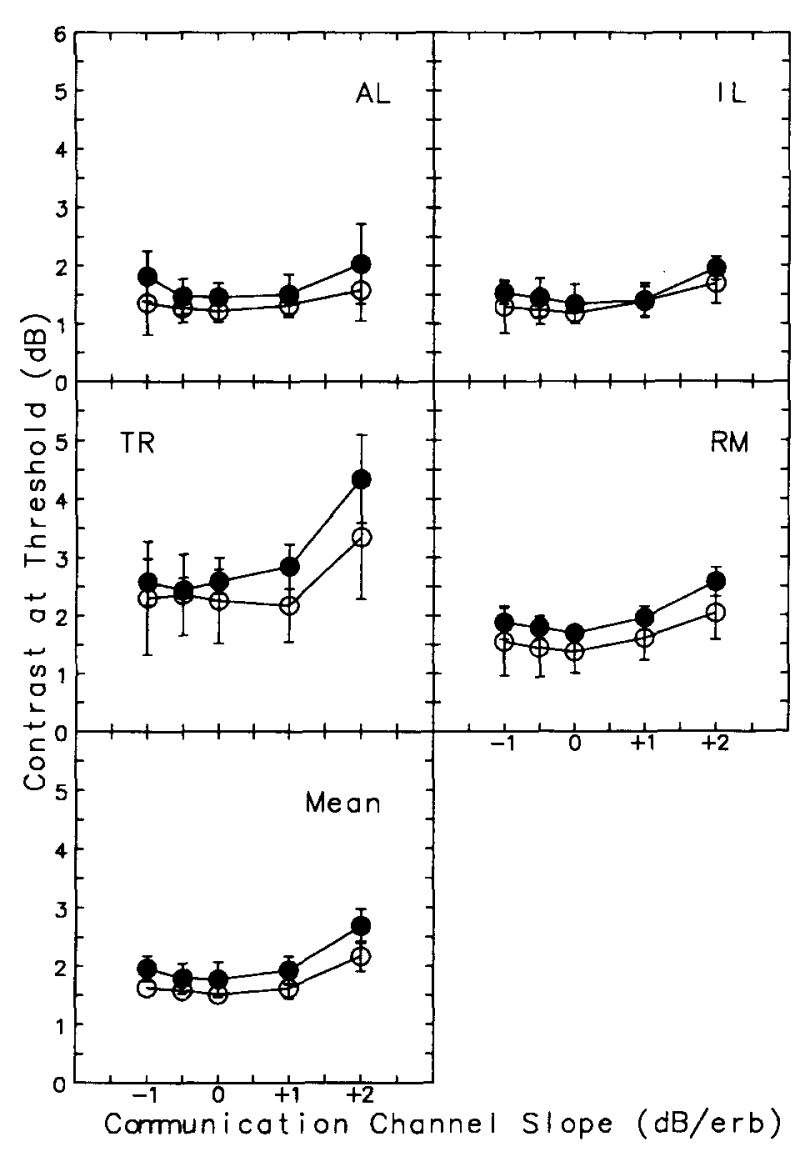

Figure 11. The filled symbols plot the results of Experiment 4 as thresholds for each listener and their mean, averaged over vowels. The abscissa shows the slope of the five communication channels. Error bars extending beyond the plotting symbol show $\pm 1 S D$ for individual listeners and $\pm 1 I L S D$ for their mean. The open symbols show the analogous results from Experiment 2 for comparison.

which thresholds were measured separately for each slope and each vowel, with slope and level fixed within and between trials.

The results of Experiments 4 and 2 were compared in a repeated measures ANOVA with the factors of experiment, slope, and vowel. Thresholds were $0.3 \mathrm{~dB}$ higher in Experiment 4 than in Experiment $2[F(1,3)=32.6$, $p<.01]$, and they varied significantly as a function of slope $[F(4,12)=10.37, p<.0007]$. There was no main effect of vowel $[F(4,12)=2.19]$ and there were no significant interactions. Post hoc tests were performed on the mean results of Experiment 4 according to the criteria recommended by Scheffé. The tests show that the communication channel slope of $+2 \mathrm{~dB} /$ erb is higher than all other communication channels (all $p \mathrm{~s}<.01$ ).

In both Experiments 2 and 4, only thresholds with the $+2-\mathrm{dB} / \mathrm{erb}$ communication channel are significantly worse than thresholds with the other four communication channels. This was not because the $+2-\mathrm{dB} / \mathrm{erb}$ com- munication channel was special, but because it had the most extreme slope, the performance of the listeners was much worse. The underlying identification threshold function must be a $U$-shaped curve. This is because with both positive and negative steeply sloping communication channels, the deterioration of frequency selectivity at high intensities (Lutfi \& Patterson, 1984; Weber, 1977 ) and the difficulties of detection near absolute threshold will cause listeners' thresholds to be high. Thus, as the communication channel slope decreases, so should the underlying identification threshold function, which should be minimal at $0 \mathrm{~dB} / \mathrm{erb}$. To show this graphically, the mean data of the 4 listeners from Experiments 2 and 4 are shown in Figure 12. Second-degree polynomials were used to fit both sets of data as they are U-shaped curves. ${ }^{2}$ As can be seen in Figure 12, the polynomials fit the sets of data closely and have minima at $0 \mathrm{~dB} / \mathrm{erb}$. Thus, it can be concluded that the thresholds from the $+2-\mathrm{dB} / \mathrm{erb}$ communication channel are only significantly and not uniquely different, as the points from each experiment lie on a curve that matches the expected underlying threshold function.

\section{Discussion}

In comparison with Experiment 2, Experiment 4 shows that random variation of level and slope within trials raises thresholds by a small but significant amount. The size of this effect does not vary significantly with vowel or slope. The outcome corroborates the results of Experiment 3 and is compatible with the idea that listeners can benefit from the strategy of spectral subtraction, but the benefits are not large.

To establish whether formants were specified by peaks or shoulders, the 25 threshold stimuli for each listener were synthesized and analyzed by using the pro-

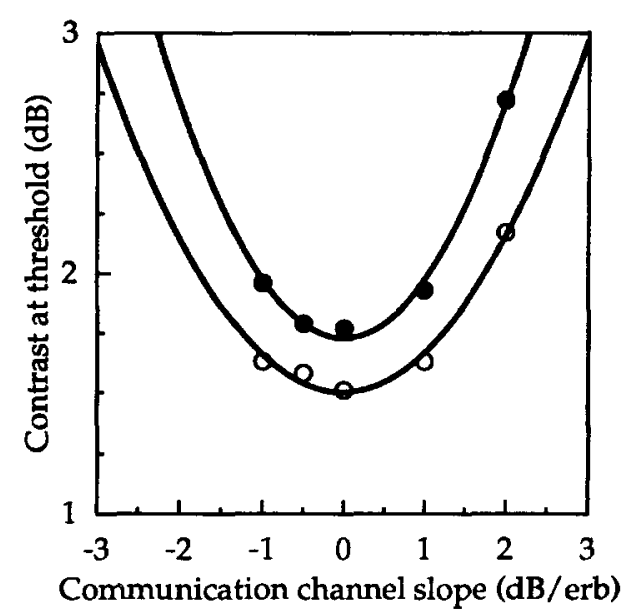

Figure 12. The filled symbols show the mean results of the 4 listeners from Experiment 4, and the open symbols show the analogous results from Experiment 2 . Both sets of data have been fitted with seconddegree polynomials, which are shown by the two curves. 
Table 3

Results of Peak and Shoulder Analysis From Experiment 4

\begin{tabular}{|c|c|c|c|c|c|}
\hline \multirow[b]{2}{*}{ Vowels } & \multicolumn{5}{|c|}{ Communication Channel Slope } \\
\hline & $-1 \mathrm{~dB} / \mathrm{erb}$ & $-0.5 \mathrm{~dB} / \mathrm{erb}$ & $0 \mathrm{~dB} / \mathrm{erb}$ & $+1 \mathrm{~dB} / \mathrm{erb}$ & $+2 \mathrm{~dB} / \mathrm{erb}$ \\
\hline \multicolumn{6}{|c|}{ Listener A.L. } \\
\hline [a] & S-- & -- & -- & $-s-$ & SSS \\
\hline [i] & --- & --. & --- & --- & SS- \\
\hline [3] & S-- & $\ldots$ & ... & --. & SSs \\
\hline [u] & -s- & --- & --- & S-s & SSS \\
\hline [o] & -- & $-\cdots$ & --- & --- & SSs \\
\hline \multicolumn{6}{|c|}{ Listener I.L. } \\
\hline [a] & S-- & --- & --- & - s- & SSS \\
\hline [i] & $--s$ & -.. & --. & --- & SSs \\
\hline [3] & S-- & --- & --- & -s- & SSs \\
\hline$[\mathrm{u}]$ & -s- & $\cdots$ & -.. & -Ss & SsS \\
\hline [॰] & $-\mathrm{Ss}$ & --- & -- & $\cdots$ & Sss \\
\hline \multicolumn{6}{|c|}{ Listener T.R. } \\
\hline [a] & --- & $-\cdots$ & $\cdots$ & $\cdots$ & $\cdots$ \\
\hline [i] & --- & --- & --- & $\cdots$ & -- \\
\hline [3] & --- & $\cdots$ & $-\cdots$ & $\cdots$ & $-\cdots$ \\
\hline$[\mathrm{u}]$ & --- & --- & --- & $\cdots$ & -- \\
\hline [0] & --- & --- & $\cdots$ & --- & -- \\
\hline \multicolumn{6}{|c|}{ Listener R.M. } \\
\hline [a] & --- & $-\ldots$ & $\ldots$ & $\cdots$ & - \\
\hline [i] & -- & -- & -- & -- & S- \\
\hline [3] & S-- & --- & --- & -.- & - \\
\hline [u] & -S- & --- & --- & --- & S-- \\
\hline [0] & -SS & --- & --- & ... & SSs \\
\hline
\end{tabular}

Peaks are indicated by "-" and shoulder by an S; an " $\mathrm{s"} \mathrm{(lowercase)} \mathrm{is}$ a shoulder that is transformed into a peak when a simple model of the effects of lateral suppression is included in the analysis. See text for details.

cedures described previously. The results are shown in Table 3. Despite the fact that it was not possible for the listeners to use the strategy of spectral subtraction, some formants of some vowels for Listeners A.L., I.L., and R.M. were specified by shoulders rather than by peaks.

In Experiment 4, the listeners were prevented from using spectral subtraction to locate the formants of target vowels. Thus, they must have identified the target vowels solely from the mixtures of targets and maskers, without reference to the isolated maskers presented in the other interval of the forced-choice procedure. Nonetheless, some formants of some vowels for some of the subjects were represented as shoulders rather than as peaks in the threshold stimuli. If Table 3 and Figure 4 are compared, it can be seen that the formants that the particular listeners used to distinguish the targets are included among those that were represented as shoulders at threshold. This outcome is compatible with Assmann and Summerfield's (1989) suggestion that to locate formants, listeners might use features of the spectrum that appear only as shoulders in excitation patterns. The conclusion confirms the weak form of the hypothesis described in the introduction: Features that appear only as shoulders in modeled excitation patterns can specify the location of formants. In the next section, we discuss the question of whether the experiments have provided evidence to support the strong form of the hypothesis - that listeners can compute the locations of formants from shoulders in their own internal spectra.

\section{EFFECTS OF PSYCHOPHYSICAL SUPPRESSION}

Excitation patterns, computed in the way described in this paper, have considerable power to explain phenomena in speech perception (e.g., Assmann \& Summerfield, 1989; Beddor \& Hawkins, 1990) and psychoacoustics (e.g., Moore \& Glasberg, 1986). However, it does not follow that they are directly equivalent to representations of signals generated within the human auditory system. One reason for an imperfect correspondence between the two is that the processes for generating excitation patterns used here omit two nonlinearities that are known to affect the shape of auditory filters. One nonlinearity is the slight increase in filter bandwidth that occurs as stimulus levels are increased (Patterson \& Moore, 1986). This effect might have reduced the spectral contrast in the more intense regions of the present stimuli, which had steeply sloping spectra. Thus, the effect of this nonlinearity would have been an increase in the number of formants represented as shoulders. However, such effects are likely to have been small at the levels at which the stimuli were presented.

The second nonlinearity is psychophysical suppression (Houtgast, 1972; Moore \& O'Loughlin, 1986). Effects of psychophysical suppression are typically demonstrated in forward masking, or in procedures similar to forward masking, where it is found that the amount of masking produced by energy at one frequency can be reduced by the presence of energy at adjacent frequencies. These effects may be related to effects seen in the auditory nerve (Arthur, Pfeiffer, \& Suga, 1971), haircells (Sellick \& Russell, 1979), and basilar membrane (Patuzzi, Sellick, \& Johnstone, 1984), wherein auditory excitation at one frequency can be reduced by the presence of more intense energy at adjacent frequencies.

Effects of psychophysical suppression are generally not shown in simultaneous masking, because both the masker and the probe signal are suppressed. Thus, the contribution of psychophysical suppression to the internal representation of the spectra of signals is considered to be revealed by a comparison of masking patterns obtained in simultaneous and forward masking. When this comparison is made by using a vowel as a masker, it is found that suppression increases the prominence of spectral peaks (Moore \& Glasberg, 1983a). The increase in spectral contrast can be as much as $15 \mathrm{~dB}$. The bandwidths of the filters used to compute excitation patterns in the present paper were determined by using simultaneous masking (Moore \& Glasberg, 1983b), and therefore they do not include effects of suppression. Potentially, therefore, a procedure for computing excitation patterns that took account of suppression might convert some shoulders in the present stimuli into peaks.

To test this idea, we used a simple simulation of the effects of suppression suggested by Moore and O'Lough- 
lin (1986). They noted that the effective enhancement of frequency selectivity produced by suppression is equivalent to reducing the bandwidths of auditory filters by $20 \%$ (e.g., Moore \& Glasberg, 1981). This change was made to the procedure used for computing excitation patterns, and the threshold stimuli from Experiment 4 were reanalyzed. Table 3 shows that although approximately $30 \%$ of the shoulders found previously were converted into peaks in this reanalysis, $70 \%$ remained as shoulders. Thus, if it is accepted that the relevant effects of suppression can be simulated by reducing the bandwidths of auditory filters, the analysis suggests that the listeners located some formants from shoulders rather than peaks in their own internal spectra.

\section{GENERAL DISCUSSION}

These experiments have shown that when a vowel has a steeply sloping spectrum, listeners can locate some formants from spectral features that appear as shoulders rather than peaks in the excitation pattern. This finding is compatible with the results of Assmann and Summerfield (1989), who found that models of vowel identification gave more accurate predictions of listeners' responses when shoulders as well as peaks were used to locate formants. However, for two reasons it would be premature to conclude that auditory analysis locates formants from shoulders in the internal auditory spectrum of signals.

First, nonlinearities in auditory analysis may convert features that appear as shoulders in the excitation patterns generated by the present model into peaks of excitation internally. Suppression is one such nonlinearity. Although the crude model described in the previous section suggested that psychophysical suppression would not convert the majority of shoulders into peaks, more sophisticated models (e.g., Patterson, Holdsworth, \& Allerhand, 1992) might convert more shoulders into peaks.

The second reason for caution in accepting the conclusion that listeners can locate formants from spectral shoulders is that a shoulder is not a robust indication of a formant in natural reverberant environments. In a reverberant environment, harmonic amplitudes are distorted by cancellation and reinforcement from echoes (e.g., Culling, Summerfield, \& Marshall, 1993), creating spurious peaks and shoulders. In such circumstances, the criterion for accepting a shoulder as evidence of a formant would have to be set considerably more conservatively than was possible here, when signals were presented quietly through headphones. Indeed, it might not be beneficial to accept any shoulder as evidence of a formant. Thus, given that shoulders normally provide unreliable evidence of formants, one can question whether auditory analysis would have developed the computational strategies for exploiting them. It may be more profitable to accept the premise that formants must be defined by peaks of excitation in auditory analysis and to use the present data as a test of the ability of models of suppression to generate such peaks.
Given these cautions, the feature of the results that should be stressed is the demonstration of a fine sensitivity to irregularities in the spectral envelope of a vowel that can define spectral locations of formants. When a vowel has a flat or gently sloping spectral envelope, listeners with normal hearing can locate a formant from a spectral peak that is defined by as little as $1.2-1.8 \mathrm{~dB}$ of contrast. This figure is close to the minimum that can be detected (Green, 1988; Henn \& Turner, 1990) and is 4 to 12 times smaller than the spectral contrast that is generally found when vowels are spoken naturally in quiet (e.g., Fant, 1960). It provides the necessary tolerance to sustain accurate vowel identification when spectral contrast is reduced by noise, reverberation, or impaired frequency selectivity.

\section{REFERENCES}

ANSI. (1969). Specifications for audiometers. New York: American National Standards Institute,

ARTHUR, R. M., PfeIfFer, R. R., \& Suga, N. (1971). Properties of twotone inhibition in primary auditory neurons. Journal of Physiology, 212, 593-609.

AssmanN, P. A., \& Summerfield, Q. (1989). Modeling the perception of concurrent vowels: Vowels with the same fundamental frequency. Journal of the Acoustical Society of America, 85, 327-338.

Assmann, P. A., \& Summerfield, Q. (1990). Modeling the perception of concurrent vowels: Vowels with different fundamental frequencies. Journal of the Acoustical Society of America, 88, 680-697.

BedDOR, P. S., \& HAWKINS, S. (1990). The influence of spectral prominence on perceived vowel quality. Journal of the Acoustical Society of America, 87, 2684-2704.

Chistovich, L. A., \& Lublinskaya, V. V. (1979). The "center of gravity" effect in vowel spectra and critical distance between the formants: Psychoacoustic study of the perception of vowel-like stimuli. Hearing Research, 1, 185-195.

Culling, J. F., Summerfield, Q., \& Marshall, D. H. (1993). Effects of simulated reverberation on the use of binaural cues and fundamental frequency differences for separating concurrent vowels. Manuscript submitted for publication.

Delattre, P., Liberman, A. M., Cooper, F. S., \& Gertsman, L. J. (1952). An experimental study of the determinants of vowel color: Observations on one- and two-formant vowels synthesized from spectrographic patterns. Word, 8, 195-210.

Dijkhuizen, J. N. van, Anema, P. C., \& Plomp, R. (1987). The effect of varying the slope of the amplitude-frequency response on the masked speech-reception threshold of sentences. Journal of the Acoustical Society of America, 81, 465-469.

FANT, G. C. M. (1960). Acoustic theory of speech production. The Hague: Mouton.

GreEN, D. M. (1988). Profile analysis: Auditory intensity discrimination. New York: Oxford University Press.

Green, D. M., KrDd, G., \& PICARDI, M. C. (1983). Successive versus simultaneous comparison in auditory intensity discrimination. Journal of the Acoustical Society of America, 73, 639-643.

HELMHOLTZ, H. L. F. voN (1968). On the physiological causes of harmony in music. In R. M. Warren \& R. P. Warren (Eds.), Helmholtz on perception: Its physiology and development. New York: Wiley. (Original work published 1863)

Henn, C. C., \& Turner, C. W. (1990). Pure tone increment detection in harmonic and inharmonic backgrounds. Journal of the Acoustical Society of America, 88, 126-131.

Houtgast, T. (1972). Psychophysical evidence for lateral suppression in hearing. Journal of the Acoustical Society of America, 51, 18851894.

Joos, M. (1948). Acoustic phonetics. Language, 24(Suppl.), 1-36. Leek, M. R., Dorman, M. F., \& Summerfield, A. Q. (1987). Mini- 
mum spectral contrast for vowel identification by normal-hearing and hearing-impaired listeners. Journal of the Acoustical Society of America, 81, 148-154.

LEVITT, H. (1971). Transformed up-down methods in psychoacoustics. Journal of the Acoustical Society of America, 49, 467-477.

LuTFI, R. A., \& PATterson, R. D. (1984). On the growth of masking asymmetry with stimulus intensity. Journal of the Acoustical Society of America, 76, 739-745.

Moore, B. C. J., \& Glasberg, B. R. (1981). Auditory filter shapes derived in simultaneous and forward masking. Journal of the Acoustical Society of America, 69, 1003-1014.

MoOre, B. C. J., \& GlasberG, B. R. (1983a). Masking patterns for synthetic vowels in simultaneous and forward masking. Journal of the Acoustical Society of America, 73, 906-917.

MoOre, B. C. J., \& Glasberg, B. R. (1983b). Suggested formulae for calculating auditory-filter shapes and excitation patterns. Journal of the Acoustical Society of America, 74, 750-753.

MOORE, B. C. J., \& GlASBERG, B. R. (1986). The role of frequency selectivity in the perception of loudness, pitch and time. In B. C. J. Moore (Ed.), Frequency selectivity in hearing (pp. 250-308.) London: Academic Press.

MoORe, B. C. J., \& GlaSBerg, B. R. (1987). Formulae describing frequency selectivity as a function of frequency and level, and their use in calculating excitation patterns. Hearing Research, 28, 209225.

MoORE, B. C. J., \& O'Loughlin, B. J. (1986). The use of nonsimultaneous masking to measure frequency selectivity and suppression. In B. C. J. Moore (Ed.), Frequency selectivity in hearing (pp. 179 249). London: Academic Press.

Patterson, R. D. (1974). Auditory filter shape. Journal of the Acoustical Society of America, 55, 802-809.

Patterson, R. D., \& Holdsworth, J. (1990). An introduction to auditory sensation processing. Cambridge, U.K.: Applied Psychology Unit.

Patterson, R. D., Holdsworth, J., \& Allerhand, M. (1992). Auditory models as preprocessors for speech recognition. In M. E. H. Schouten (Ed.), The auditory processing of speech: From the auditory periphery to words (pp. 67-83). Berlin: Mouton de Gruyter.

Patterson, R. D., \& MoORE, B. C. J. (1986). Auditory filters and excitation patterns as representations of frequency resolution. In
B. C. J. Moore (Ed.), Frequency selectivity in hearing (pp. 123-177). London: Academic Press.

Patuzzi, R., Sellick, P. M., \& Johnstone, B. M. (1984). The modulation of the sensitivity of the mammalian cochlear by low frequency tones: III. Basilar membrane motion. Hearing Research, 13, 19-27.

Sellick, P. M., \& Russell, I. J. (1979). Two-tone suppression in cochlear hair cells. Hearing Research, 1, 227-236.

Summerfield, Q., \& AssmanN, P. F. (1991). Perception of concurrent vowels: Effects of harmonic misalignment and pitch-period asynchrony. Journal of the Acoustical Society of America, 89, 13641377.

Summerfield, A. Q., SidWell, A., \& Nelson, T. (1987). Auditory enhancement of changes in spectral amplitude. Journal of the Acoustical Society of America, 81, 700-708.

Turner, C. W., \& VAN TASEll, D. J. (1984). Sensorineural hearing loss and the discrimination of vowel-like stimuli. Journal of the Acoustical Society of America, 75, 562-565.

WEBER, D. L. (1977). Growth of masking and the auditory filter. Journal of the Acoustical Society of America, 62, 424-429.

WINER, B. J. (1971). Statistical principles in experimental design (2nd ed.). London: McGraw-Hill.

ZWicker, E., \& FeldTKeller, R. (1967). Das Ohr als Nachrichtenempfänger. Stuttgart: Hirzel Verlag.

\section{NOTES}

1. The fact that the formant is boosted by only $2.9 \mathrm{~dB}$ after summing with the masker without attenuation can be understood by considering the previously defined formula: $s=20 \log _{10}\left(10^{(\mathrm{r} / 20)}+1\right)$, where $s$ is the relative level of the two sinusoids of the same frequency and phase, one of which has a relative level of $0 \mathrm{~dB}$ and the other $r \mathrm{~dB}$. If $r$ is $0 \mathrm{~dB}$, $s$ is $6.02 \mathrm{~dB}$. If $r$ is boosted by $5 \mathrm{~dB}, s$ is $8.88 \mathrm{~dB}$. Thus, the sum is boosted by $2.86 \mathrm{~dB}(=8.88-6.02)$.

2. The polynomial fitted to the data from Experiment 2 is $f(x)=$ $1.62 E^{-1} x^{2}+3.35 E^{-3} x+1.50$. The polynomial fitted to the data from Experiment 4 is $f(x)=2.45 E^{-1} x^{2}-1.02 E^{-3} x+1.73$.

(Manuscript received May 20, 1993; revision accepted for publication March 2, 1994.) 